\title{
Tetrapyrrole Singlet Excited State Quenching by Carotenoids in an Artificial Photosynthetic Antenna
}

\author{
Rodrigo E. Palacios, Gerdenis Kodis, Cristian Herrero, Ernesto Mariño Ochoa, Miguel \\ Gervaldo, Stephanie L. Gould, John T. M. Kennis, Devens Gust, * Thomas A. Moore, * \\ and Ana L. Moore*
}

Calculation of $\Delta G_{\mathrm{p}}^{\mathbf{0}}$ Using the FDPB Method. Equation 6 of the paper was used to calculate the $\Delta G_{j}^{m}$ values necessary for the estimation of $k_{j}^{m}$. In this case the electron transfer process of interest is the one initiated by photoexcitation of the phthalocyanine moiety in triads $\mathbf{1}$ and $\mathbf{2}$ which leads to the formation of a $\mathrm{C}^{{ }^{+}-}-\mathrm{Pc}^{{ }^{-}-}$charge transfer state. Consequently, the carotenoid and phthalocyanine moieties of the triads represent the donor and acceptor entities, respectively. $E_{\mathrm{Ox}}^{D}$ and $E_{\mathrm{Red}}^{A}$ are their corresponding oxidation and reduction potentials (as listed in Table 1 of the paper), and $E_{00}$ is the energy of the $\operatorname{Pc}\left(\mathrm{S}_{1}\right)$ state given in Table 2 of the paper, for the different solvents studied. The $C$ and $S$ terms in Equation 6 of the paper were calculated using the DelPhi v.4 program ${ }^{1-5}$. For each set of calculations the following input files are required: coord.pdb (molecule's atomic coordinates), initial/final.crg (initial/final charge distribution), size.siz, and parameters.prm (calculation parameters). The coord.pdb files were created using the previously described three-dimensional structure of triads $\mathbf{1}$ and $\mathbf{2}$. In order to construct the initial.crg files, these coordinates were used, and the charge on each atomic site $(q(n))$ 
was obtained from (single point) density functional theory (B3LYP) calculations with a 3-21G basis set and the Merz-Kollman charge fitting scheme. ${ }^{6,7}$ For the calculation of the final.crg files, it is necessary to compute the change in the initial charge distribution $(\Delta q(\mathrm{n}))$. In order to estimate this change, models for Pc and $\mathrm{C}$ moieties were constructed for each triad. The phthalocyanine models were built from the triad's three-dimensional structure by replacing the polyene chains from the first carbon after the carbonyl group (C9' and C7' for $\mathbf{1}$ and $\mathbf{2}$, respectively) with a methyl group. In a similar way, the C models were created by taking the coordinates of the corresponding s-trans carotenoid moieties in $\mathbf{1}$ and $\mathbf{2}$, and replacing the rest of the molecule (starting from the silicon atom) by a $\mathrm{Si}\left(\mathrm{CH}_{3}\right)_{3}$ group. Subsequently, $\Delta q(\mathrm{n})$ for each triad was computed by combining the charge difference between the "charged" $\left(\mathrm{Pc}^{\bullet-} / \mathrm{C}^{\bullet+}\right)$ and the "neutral" charge distribution of the corresponding models ${ }^{8}$. In order to illustrate the outcome of the procedure, the resulting change of charge distribution is shown in Figure 1 panel A and B, for triads 2 and 1, respectively. Finally, the calculated $\Delta q(\mathrm{n})$ was added to the corresponding $q(\mathrm{n})$ to create the final.crg file for each triad.

The size.siz files were created using the van der Waals radius for the atoms in the triads. Parameters.prn files, specifying solvent radius ${ }^{9}$ and dielectric constant, were created for each solvent using the information in Table 1. Additionally, to account for the electronic polarizability of the solute the dielectric constant inside the triads' cavity $\left(\varepsilon_{\mathrm{IN}}\right)$ was set to 2.0 .

Table 1. Solvent Parameters used in the FDPB calculations

\begin{tabular}{cccc}
\hline Solvent & $\begin{array}{c}\text { Hard sphere } \\
\text { solvent radius }\end{array}$ & $\begin{array}{c}\text { Static } \\
\text { dielectric constant }\end{array}{ }^{d}$ & $\begin{array}{c}\text { High frequency } \\
\text { dielectric constant }^{e}\end{array}$ \\
\hline$n$-hexane & $2.96^{a}$ & 1.88 & 1.88 \\
toluene & $2.83^{b}$ & 2.38 & 2.24 \\
tetrahydrofuran & $2.50^{c}$ & 7.52 & 1.97
\end{tabular}


benzonitrile

${ }^{a}$ Calculated as an average of the values given in ref $10 .{ }^{b}$ From ref $11 .{ }^{c}$ From ref $12 .{ }^{d}$ From ref 13. ${ }^{e}$ Calculated from the relationship $\varepsilon_{\infty}=n^{2}$ and data from ref 14 .

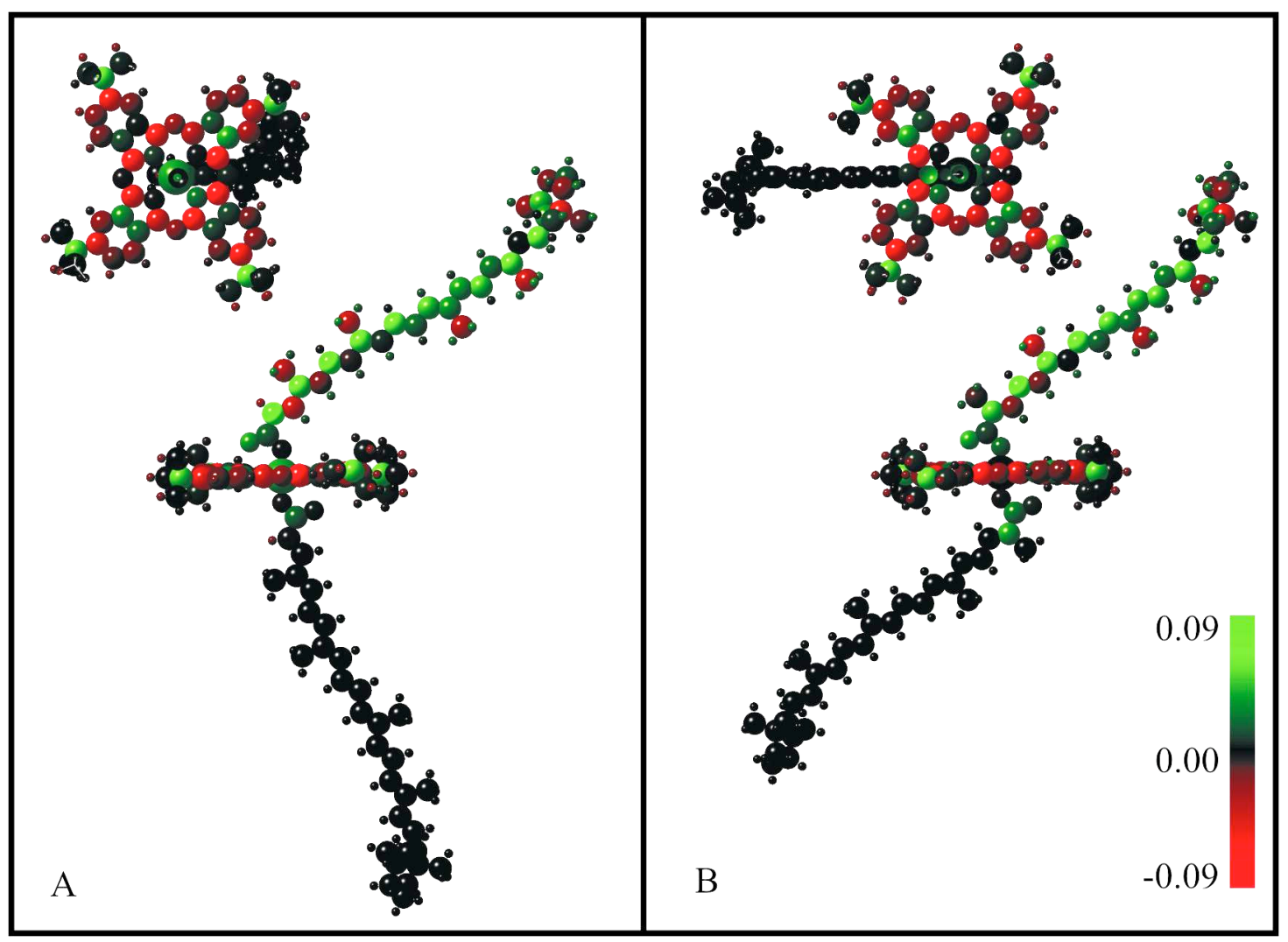

Figure 1 (A) Three dimensional structure of triad 2 showing the change in charge-distribution upon electron transfer. The color-scale is based on the charge of an electron. (B) Same as (A) for triad $\mathbf{1 .}$

After the calculations are concluded the program generates an output file containing Coulomb and solvation energies. In this context, the Coulomb energy $(C)$ is defined as the energy required to bring charges from infinite distance to their resting positions within the dielectric specified for the molecule, and the solvation energy $(S)$ is the energy of transferring the molecule from a medium equal to the interior dielectric of the molecule $\left(\varepsilon_{\mathrm{IN}}\right)$ into a medium of external dielectric of the solution. Based on these 
definitions and considering Equation 6 of the paper, the electron transfer driving force in benzonitrile $\left(\left(\Delta G_{\mathrm{P}}^{\mathrm{o}}\right)_{\mathrm{PhCN}}\right)$ was calculated using the following thermodynamic cycle.

$$
\begin{aligned}
& 2(D-A)_{\mathrm{PhCN}} \rightarrow\left(D^{*+}-A\right)_{\mathrm{PhCN}}+\left(D-A^{*-}\right) \mathrm{PhCN} \\
& \left(D^{\circ+}-A\right)_{\mathrm{PhCN}} \rightarrow\left(D^{\cdot+}-A\right)_{\mathrm{IN}} \\
& \left(D^{\circ+}-A\right)_{\mathrm{IN}} \rightarrow(D-A)_{\mathrm{IN}} \\
& \left(D-A^{\cdot-}\right)_{\mathrm{PhCN}} \rightarrow\left(D-A^{\bullet-}\right)_{\mathrm{IN}} \\
& \left(D-A^{\cdot-}\right)_{\mathrm{IN}} \rightarrow(D-A)_{\mathrm{IN}} \\
& (D-A)_{\mathrm{IN}} \rightarrow\left(D^{\cdot+}-A^{\bullet-}\right)_{\mathrm{IN}} \\
& (D-A)_{\mathrm{IN}} \rightarrow(D-A)_{\mathrm{PhCN}} \\
& \left(D^{{ }^{+}-} A^{\cdot-}\right)_{\mathrm{IN}} \rightarrow\left(D^{\cdot+}-A^{\cdot-}\right)_{\mathrm{PhCN}} \\
& \left({ }^{1} D-A\right)_{\mathrm{PhCN}} \rightarrow(D-A)_{\mathrm{PhCN}} \\
& \left({ }^{1} D-A\right)_{\mathrm{PhCN}} \rightarrow\left(D^{\cdot+}-A^{\cdot-}\right)_{\mathrm{PhCN}} \\
& \left(E_{\mathrm{Ox}}^{D A}\right)_{\mathrm{PhCN}}-\left(E_{\mathrm{Red}}^{D A}\right)_{\mathrm{PhCN}} \\
& -\left(S_{D^{*+} A}\right)_{\mathrm{PhCN}} \\
& C_{D A}-C_{D^{{ }^{+} A}} \\
& -\left(S_{D^{*+} A}\right)_{\mathrm{PhCN}} \\
& C_{D A}-C_{D A^{-*}} \\
& C_{D^{*+A^{-0^{-}}}}-C_{D A} \\
& \left(S_{D A}\right)_{\mathrm{PhCN}} \\
& \left(S_{D^{*+} A^{\cdot-}}\right)_{\mathrm{PhCN}} \\
& -\left(E_{00}\right)_{\mathrm{PhCN}} \\
& \left(\ddot{\mathrm{A}} G_{\mathrm{p}}^{\mathrm{o}}\right)_{\mathrm{PhCN}}=\left(E_{\mathrm{Ox}}^{D A}\right)_{\mathrm{PhCN}}-\left(E_{\mathrm{Red}}^{D A}\right)_{\mathrm{PhCN}}+ \\
& +\left(C_{D^{*^{+} A^{*-}}}+C_{D A}\right)-\left(C_{D^{D^{*+} A}}+C_{D A^{*^{-}}}\right)+ \\
& +\left[\left(S_{D^{+*} A^{-*}}\right)_{\mathrm{PhCN}}+\left(S_{D A}\right)_{\mathrm{PhCN}}\right]- \\
& -\left[\left(S_{D^{*+} A}\right)_{\mathrm{PhCN}}+\left(S_{D A^{*-}}\right)_{\mathrm{PhCN}}\right]-\left(E_{00}\right)_{\mathrm{PhCN}}
\end{aligned}
$$

where $D^{\bullet^{*}-\mathrm{A}^{*-}}$ and $D-A$ refer to the initial and final charged separated states, respectively, for each triad.

Once $\left(\ddot{\mathrm{A}} G_{\mathrm{p}}^{\mathrm{o}}\right)_{\mathrm{PhCN}}$ is obtained, the diving force in any other solvent $\left(\ddot{\mathrm{A}} G_{\mathrm{p}}^{\mathrm{o}}\right)_{\text {Solv }}$ can be calculated using following cycle.

$$
\begin{aligned}
& \left({ }^{1} D-A\right)_{\mathrm{PhCN}} \rightarrow\left(D^{*+}-A^{*-}\right)_{\mathrm{PhCN}} \\
& \left(\Delta G_{\mathrm{P}}^{\mathrm{o}}\right)_{\mathrm{PhCN}} \\
& \left(D^{\cdot+}-A^{\cdot-}\right)_{\mathrm{PhCN}} \rightarrow\left(D^{\cdot+}-A^{\cdot-}\right)_{\mathrm{IN}} \\
& (D-A)_{\mathrm{PhCN}} \rightarrow\left({ }^{1} D-A\right)_{\mathrm{PhCN}} \\
& (D-A)_{\mathrm{IN}} \rightarrow(D-A)_{\mathrm{PhCN}} \\
& -\left(S_{D^{+*} A^{*-}}\right)_{\mathrm{PhCN}} \\
& (D-A)_{\mathrm{Solv}} \rightarrow(D-A)_{\mathrm{IN}} \\
& \left(\mathrm{E}_{00}\right)_{\mathrm{PhCN}} \\
& \left(S_{D A}\right)_{\mathrm{PhCN}} \\
& \left({ }^{1} D-A\right)_{\text {Solv }} \rightarrow(D-A)_{\text {Solv }} \\
& -\left(S_{D A}\right)_{\text {Solv }} \\
& \left(D^{\bullet+}-A^{\bullet-}\right)_{\mathrm{IN}} \rightarrow\left(D^{*^{+}-} A^{\cdot-}\right)_{\text {Solv }} \\
& -\left(E_{00}\right)_{\text {Solv }} \\
& \left(S_{D^{+A^{*}}}\right)_{\text {Solv }}
\end{aligned}
$$




$$
\begin{aligned}
\left({ }^{1} D-A\right)_{\mathrm{Solv}} \rightarrow\left(D^{\cdot+}-A^{\cdot-}\right)_{\mathrm{Solv}} & \left(\ddot{\mathrm{A}} G_{\mathrm{p}}^{\mathrm{o}}\right)_{\mathrm{Solv}}=\left(\ddot{\mathrm{A}} G_{\mathrm{p}}^{\mathrm{o}}\right)_{\mathrm{PhCN}}+\left[\left(E_{00}\right)_{\mathrm{PhCN}}-\right. \\
& \left.-\left(E_{00}\right)_{\mathrm{Solv}}\right]+\left[\left(S_{D^{*+} A^{-\cdot}}\right)_{\mathrm{Solv}}-\left(S_{D A}\right)_{\mathrm{Solv}}\right]+ \\
& +\left[\left(S_{D A}\right)_{\mathrm{PhCN}}-\left(S_{D^{+*} A^{--}}\right)_{\mathrm{PhCN}}\right]
\end{aligned}
$$

Calculation of $\lambda_{\mathrm{p}}$ Using the FDPB Method. Considering the relation given by Equation 7 of the paper, the $\lambda_{j}^{m}$ values where computed as the difference between the solvation energies of the charge-difference distribution, $\Delta q(\mathrm{n})$ (Figure 1), calculated using the static and optical dielectric constants of the corresponding solvent.

The results of the previously described $\Delta G_{\mathrm{p}}^{\mathrm{o}}$ and $\lambda_{\mathrm{p}}$ calculations are summarized in Table 2.

Table 2: Calculated driving forces and reorganization energies at room temperature.

\begin{tabular}{ccccc}
\hline & \multicolumn{2}{c}{ Triad 2 } & \multicolumn{2}{c}{ Triad 1 } \\
Solvent & $\Delta G_{\mathrm{p}}^{\mathrm{o}}$ & $\lambda_{\mathrm{p}}$ & $\Delta G_{\mathrm{p}}^{\mathrm{o}}$ & $\lambda_{\mathrm{p}}$ \\
\hline$n$-hexane & 0.169 & 0.000 & 0.245 & 0.000 \\
Toluene & 0.049 & 0.031 & 0.135 & 0.030 \\
Tetrahydrofuran & -0.387 & 0.462 & -0.273 & 0.459 \\
Benzonitrile & -0.503 & 0.502 & -0.377 & 0.492
\end{tabular}

\section{References}

(1) Nicholls, A.; Honig, B. J. Comput. Chem. 1991, 12, 435-445.

(2) Rocchia, W.; Alexov, E.; Honig, B. J. Phys. Chem. B 2001, 105, 6754.

(3) Rocchia, W.; Sridharan, S.; Nicholls, A.; Alexov, E.; Chiabrera, A.; Honig, B. J. Comput. Chem. 2002, 23, 128-137.

(4) Sharp, K.; Honig, B. Chemica Scripta 1989, 29A, 71-74.

(5) Sharp, K. A.; Honig, B. Annual Review of Biophysics and Biophysical Chemistry 1990, 19, 301-332. 
(6) Besler, B. H.; Merz, K. M., Jr.; Kollman, P. A. J. Comput. Chem. 1990, 11, 431439.

(7) Singh, U. C.; Kollman, P. A. J. Comput. Chem. 1984, 5, 129-145.

(8) The "charged" and "neutral" distributions were calculated using the previously described quantum chemical method. The charge in the extra atoms of the models compared to the triads was distributed uniformly on each moiety.

(9) The program generates the boundary between the solute and solvent by rolling a probe solvent molecule (approximated by a sphere) along the van der Waals surface of the solute. All points inaccessible to the probe sphere's surface are considered to belong to the solute.

(10) Gogonea, V.; Baleanu-Gogonea, C.; Osawa, E. THEOCHEM 1998, 432, 177-189.

(11) Read, I.; Napper, A.; Zimmt, M. B.; Waldeck, D. H. J. Phys. Chem. A 2000, 104, 9385-9394.

(12) Kumar, K.; Kurnikov, I. V.; Beratan, D. N.; Waldeck, D. H.; Zimmt, M. B. J. Phys. Chem. A 1998, 102, 5529-5541.

(13) Wohlfarth, C. Static Dielectric Constants of Pure Liquids and Binary Liquid Mixtures; Springer-Verlag: Berlin, Heidelberg, New York, 1991; Vol. 6.

(14) Lide, D. R. CRC Handbook of Chemistry and Physics, 84th ed.; CRC Press LLC: Boca Raton, 2003. 